\title{
Hyperinflammatory Syndrome in Children Associated With COVID-19: Need for Awareness
}

\author{
Chandrika S Bhat, ${ }^{1}$ Latika Gupta, ${ }^{2}$ S Balasubramanian, ${ }^{3}$ SurJit Singh ${ }^{4}$ and Athimalaipet V Ramanan ${ }^{5}$ \\ From ${ }^{1}$ Pediatric Rheumatology Service, Rainbow Children's Hospital, Bangalore, Karnataka, India; ${ }^{2}$ Department of Clinical \\ Immunology and Rheumatology, Sanjay Gandhi Postgraduate Institute of Medical Sciences, Lucknow, Uttar Pradesh India; \\ ${ }^{3}$ Department of Pediatrics, Kanchi Kamakoti CHILDS Trust Hospital, Chennai, Tamil Nadu, India; ${ }^{4}$ Allergy Immunology Unit, \\ Department of Pediatrics, Advanced Pediatrics Centre, Post Graduate Institute of Medical Education and Research, Chandigarh, \\ India; and ${ }^{5}$ Bristol Royal Hospital for Children and Translational Health Sciences, University of Bristol, Bristol, UK. \\ Correspondence: Dr Chandrika S Bhat, Rainbow Children's Hospital, Marathahalli, Bengaluru 560 037, Karnataka, India.
}

The pandemic of COVID-19 initially appeared to cause only a mild illness in children. However, it is now apparent that a small percentage of children can develop a hyperinflammatory syndrome labeled as Pediatric inflammatory multisystem syndrome - temporally associated with SARS-CoV-2 (PIMS-TS). Features of this newly recognized condition may include persistent fever, evidence of inflammation, and single or multi-organ dysfunction in the absence of other known infections. Some of these children may share features of Kawasaki disease, toxic shock syndrome or cytokine storm syndrome. They can deteriorate rapidly and may need intensive care support as well. The PCR test is more often negative; although, most of the children have antibodies to SARS-CoV-2. Although the pathogenesis is not clearly known, immune-mediated injury has been implicated. We herein provide current information on this condition, in order to raise awareness amongst pediatricians.

Keywords: Kawasaki disease, Macrophage activation syndrome, Multisystem inflammatory syndrome in children and adolescents temporally related to COVID-19, Pediatric inflammatory multisystem syndrome - temporally associated with SARS-CoV-2 (PIMS-TS).

Published online: July 15, 2020; PII: S097475591600208

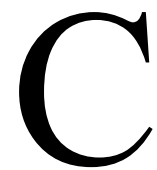
hildren younger than 18 years have been reported to constitute only a small proportion of cases of coronavirus disease (COVID-19). Whilst initial reports described an asymptomatic or milder illness in children [1,2], several countries have now noticed a new hyper-inflammatory syndrome affecting a small percentage of children [3]. This condition appears to share features with pediatric inflammatory diseases such as Kawasaki disease (KD) and Toxic shock syndrome (TSS) [4].

The first case of classic KD with concurrent COVID19 in a child was reported from United States [5]. Subsequently, health authorities in the United Kingdom (UK) issued an alert describing a serious illness requiring intensive care in children. A number of other regions significantly affected by COVID-19 such as New York, Italy and France also reported increasing numbers of children with a similar inflammatory syndrome [3]; the first such case was reported from India only recently [6]. The Royal College of Pediatrics and Child Health (RCPCH) published a guidance to raise awareness amongst clinicians for this newly recognized condition called Pediatric inflammatory multisystem syndrome temporally associated with SARS-CoV-2 (PIMS-TS) [4]. A similar clinical entity was defined as the Multisystem inflammatory syndrome in children and adolescents temporally related to COVID-19 by the World Health Organization (WHO) [7] and Multisystem inflammatory syndrome in children (MIS-C) associated with COVID19 [8] by Centers for Disease Control and prevention (CDC) (Box I). Although little is known about the epidemiology, cases of PIMS-TS seem to appear few weeks after the COVID-19 peak in the population. As of 13 May, 2020, there were more than 300 cases of suspected PIMS-TS in Europe and North America [3]. With India lagging behind the peak curve, the authors hypothesize that we may also see a spurt in this illness in the coming days.

\section{CLINICAL FEATURES}

One of the initial reports [9] described a cluster of eight children with hyperinflammatory shock. Mean age at presentation was 8.8 years with a predilection for boys of Afro-Caribbean descent and seven of these were above the $75^{\text {th }}$ centile for weight. Mean duration of fever at presentation was 4.3 days. Mucocutaneous changes (rash, conjunctivitis, peripheral edema) with significant gastrointestinal symptoms were noted in all of them. All 8 patients developed severe refractory shock with a mean ferritin level of $1086.6 \mathrm{ng} / \mathrm{mL}$. One child required extra- 
Box I Proposed Case Definitions for the Hyperinflammatory Syndrome Associated With COVID-19 [4,7,8] World Health Organization

Children and adolescents 0-19 years of age with fever $>3$ days

AND two of the following:

(a) Rash or bilateral non-purulent conjunctivitis or muco-cutaneous inflammation signs (oral, hands or feet)

(b) Hypotension or shock

(c) Features of myocardial dysfunction, pericarditis, valvulitis, or coronary abnormalities (including $\mathrm{ECHO}$ findings or elevated Troponin/NT proBNP)

(d) Evidence of coagulopathy (by PT, PTT, elevated D-dimer)

(e) Acute gastrointestinal problems (diarrhea, vomiting, or abdominal pain)

AND

Elevated markers of inflammation such as ESR, CRP or procalcitonin.

AND

No other obvious microbial cause of inflammation, including bacterial sepsis, staphylococcal or streptococcal shock syndromes.

AND

Evidence of COVID-19 (RT-PCR, antigen test or serology positive), or likely contact with patients with COVID-19. Royal College of Pediatrics and Child Health

A child presenting with persistent fever, inflammation (neutrophilia, elevated CRP and lymphopenia) and evidence of single or multi-organ dysfunction.

This may include children fulfilling full or partial criteria for Kawasaki disease.

Exclusion of any other microbial cause, including bacterial sepsis, staphylococcal or streptococcal shock syndromes, infections associated with myocarditis such as enterovirus.

SARS-CoV-2 PCR testing may be positive or negative.

\section{Centers for Disease Control}

An individual aged $<21$ years presenting with fever, laboratory evidence of inflammation and evidence of clinically severe illness requiring hospitalization, with multisystem $(\geq 2)$ organ involvement (cardiac, renal, respiratory, hematologic, gastrointestinal, dermatologic or neurological)

(i) Fever $\geq 38.0^{\circ} \mathrm{C}$ for $\geq 24$ hours, or report of subjective fever lasting $\geq 24$ hours.

(ii) Laboratory evidence (but not limited to) of one or more of the following: an elevated CRP, ESR, fibrinogen, procalcitonin, D-dimer, ferritin, LDH, or interleukin 6, elevated neutrophils, reduced lymphocytes and low albumin.

AND

No alternative plausible diagnoses

AND

Positive for current or recent SARS-CoV-2 infection by RT-PCR, serology, or antigen test; Or COVID-19 exposure within 4 weeks prior to the onset of symptoms.

CRP: C-reactive protein; ESR: Erythrocyte sedimentation rate; $L D H$ : Lactate dehydrogenase.

corporeal membrane oxygenation (ECMO) for refractory shock but eventually died after 6 days of hospitalization. None of the children had respiratory symptoms and only two tested positive for SARS-CoV-2 PCR, while all of them tested positive for the antibody [9]. Ten children presenting with features of classic or incomplete KD were reported from Italy [10] with mean age and duration of fever of 7.5 years and 6 days, respectively. Apart from gastrointestinal and mucocutaneous symptoms, meningeal signs were also reported in this subset. Half of them developed KD shock syndrome (KDSS) with peak ferritin levels of $1176 \mathrm{ng} / \mathrm{mL}$. In comparison to children 
with $\mathrm{KD}$ in pre-pandemic times the current phenotype included older children with more severe disease, significant cardiac involvement and macrophage activation syndrome (MAS) [10]. Again, only two tested positive for SARS-CoV-2 PCR, but eight tested positive for the antibody. In both the groups, inflammatory markers (C-reactive protein, procalcitonin, ferritin, triglycerides, and D-dimer) were significantly elevated. An abnormal echocardiogram with myocardial dysfunction and coronary artery abnormalities were observed in $60 \%$ children, and two also had coronary aneurysms [10].

More recently, a French study [11] described a new syndrome complex of acute heart failure and hyperinflammation in children. Initial presentation predominantly included fever $(100 \%)$ and gastro-intestinal symptoms (80\%) such as abdominal pain, vomiting and diarrhea. Although mucocutaneous changes suggestive of KD were noted, none of them met the criteria for classic KD. Echocardiography was significant for left ventricular dysfunction with a low ejection fraction. Inflammatory markers (CRP, D-dimer) were raised in all. Coronary artery dilatation was seen in $17 \%$, but as opposed to classic KD, none of them developed coronary aneurysms. Complete recovery was seen in $71 \%$ of children, suggesting that myocardial edema rather than necrosis was likely responsible for heart failure. This is in contrast to the adult population, where myocardial necrosis has been incriminated in the pathogenesis [11].

The importance of suspecting PIMS-TS in febrile adolescent children with gastrointestinal symptoms during this pandemic cannot be overemphasized. This unusual presentation was also reinforced in a case series of eight children from UK, initially suspected to have appendicitis [12]. Although they had very high CRP levels, abdominal imaging demonstrated non-specific features (e.g. lymphadenopathy or ileitis) rather than appendicitis. Subsequently, half of these children required intensive care admission for hemodynamic instability. Apart from peripheral or periorbital edema, none of them had features to suggest classic KD and five tested positive for SARS-CoV-2 [12].

In a larger case series of 58 children (median age 9 years) from UK [13], all presented with fever and combinations of abdominal pain (53\%), diarrhea (52\%) or rash $(52 \%)$. Three clinical patterns were identified in this cohort- fever with raised inflammatory markers (39.6\%) without features of KD, TSS or organ failure; shock $(50 \%)$ with evidence of left ventricular dysfunction $(62 \%)$; and those fulfilling criteria for KD. Coronary artery aneurysms were noted across all three groups (8/ 58). Compared to other inflammatory disorders, those with PIMS-TS were older and had lower hemoglobin levels and lymphocyte counts, and higher white blood cell count, neutrophil count and CRP levels (Table I) [13].

It appears that these children may develop single or multi-organ dysfunction with persistent fever and features of inflammation (neutrophilia, elevated CRP and lymphopenia). This may progress on to shock. In patients who turn out to be SARS-CoV-2 PCR negative, other microbial causes need to be actively considered and excluded [4]. In addition to KD and TSS, secondary hemophagocytic lymphohistiocytosis (HLH) in association with common tropical infections should also be considered in similar clinical settings. Based on available data, we speculate that there could be three distinct phenotypes of hyperinflammation in children (Table II).

\section{PATHOGENESIS}

Approximately two-thirds of patients with PIMS-TS are COVID-19 PCR negative, a proportion of these being serologically positive, suggesting an immune-mediated

Table I Comparison of PIMS-TS With Classic KD, KDSS and TSS [13]

\begin{tabular}{lllll}
\hline Features & $P I M S-T S(n=58)$ & $K D(n=1132)$ & KDSS $(n=45)$ & TSS $(n=46)$ \\
\hline Age at onset, y & $9.0(5.7-14)$ & $2.7(1.4-4.7)$ & $3.8(0.2-18)$ & $7.38(2.4-15.4)$ \\
CRP, mg/L & $229(156-338)$ & $67(40-150)$ & $193(83-237)$ & $201(122-317)$ \\
Hemoglobin, g/L & $92(83-103)$ & $111(105-119)$ & $107(98-115)$ & $114(98-130)$ \\
Lymphocytes, $\times 10^{9} / \mathrm{L}$ & $0.8(0.5-1.5)$ & $2.8(1.5-4.4)$ & $1.6(1-2.5)$ & $0.63(0.41-1.13)$ \\
Ferritin, $\mu \mathrm{g} / \mathrm{L}$ & $610(359-1280)$ & $200(143-243)$ & $301(228-337)$ & - \\
NT-Pro-BNP, pg/mL & $788(174-10548)$ & $41(12-102)$ & $396(57-1520)$ & - \\
Troponin, ng/L & $45(8-294)$ & $10(10-20)$ & $10(10-30)$ & - \\
D-dimer, ng/mL & $3578(2085-8235)$ & $1650(970-2660)$ & $2580(1460-2990)$ & \\
\hline
\end{tabular}

Data are median (IQR); PIMS-TS: pediatric inflammatory multisystem syndrome-temporally related to SARS-CoV-2, KD: Kawasaki disease, KDSS: Kawasaki disease shock syndrome, TSS: Toxic shock syndrome, CRP: C-reactive protein. 
Table II Possible Phenotypes of SARS-CoV-2-Related Hyperinflammation in Children [4,18, 24]

\begin{tabular}{|c|c|c|}
\hline Classic Kawasaki disease & $\begin{array}{l}\text { Pediatric inflammatory multisystem } \\
\text { syndrome-temporally associated } \\
\text { with } S A R S-C o V-2\end{array}$ & Macrophage activation syndrome \\
\hline \multicolumn{3}{|l|}{ Clinical features } \\
\hline - Younger children $(<5 \mathrm{y})^{*}$ & - Older children and adolescents & - Adolescents \\
\hline - Fever (usually $>5$ d) with any $4 / 5$ : & - Fever with: & - Unremitting fever \\
\hline - Non purulent conjunctivitis & - Gastrointestinal symptoms & - Pulmonary involvement \\
\hline - Cervical lymphadenopathy $>1.5 \mathrm{~cm}$ & - Mucocutanoeus changes & - Organomegaly(Hepatosplenomegaly) \\
\hline - Erythematous rash & - Confusion or headache & \\
\hline - Mucositis- strawberry tongue & - Single or multisystem dysfunction. & \\
\hline - Extremity changes- swelling/peeling & - Rapid deterioration with refractory & \\
\hline $\begin{array}{l}\text { High incidence of coronary artery } \\
\text { aneurysms. } \\
\text { - Refractory to therapy }\end{array}$ & shock. & \\
\hline \multicolumn{3}{|l|}{ Laboratory markers } \\
\hline - $\mathrm{CRP} \geq 3.0 \mathrm{mg} / \mathrm{dL}$ and $/$ or $\mathrm{ESR} \geq 40 \mathrm{~mm} / \mathrm{h}$ & - High CRP & - Cytopenia (at least 2 cell lines affected) \\
\hline - Elevated ALT & - Lymphopenia & - Hypertriglyceridemia \\
\hline - Albumin $\leq 3.0 \mathrm{~g} / \mathrm{dL}$ & - Neutrophilia in most & - Hypofibrinogenemia \\
\hline - $\mathrm{WBC}>15,000$ & - Abnormal fibrinogen & - High ferritin \\
\hline - Anemia for age & - High D-Dimers & - High AST \\
\hline - Platelets $>450000$ ( $>7 \mathrm{~d}$ of fever) & - High ferritin & - Haemophagocytosis on bone marrow \\
\hline - Urine analysis- $10 \mathrm{WBCs}$ per high & - Raised LDH & aspirate \\
\hline power field & - Hypoalbuminemia & - Low or absent NK cell activity \\
\hline & - Transaminitis & - Elevated soluble CD25 levels \\
\hline & - Elevated troponin, NT-proBNP & \\
\hline \multicolumn{3}{|l|}{ Echocardiogram } \\
\hline \multirow[t]{5}{*}{ - Coronary artery dilatation or aneurysms. } & - Left ventricular dysfunction & - Left ventricular dysfunction \\
\hline & - Myocarditis & - Coronary artery dilatation or aneurysm \\
\hline & - Valvulitis & \\
\hline & - Pericardial effusion & \\
\hline & - Coronary artery dilatation & \\
\hline
\end{tabular}

*We believe that children under 1 year of age are at particular risk of coronary aneurysms in KD seen in COVID era (unpublished data from authors). CRP: C-reactive protein; ESR: erythrocyte sedimentation rate; WBC: white blood cell; AST: aspartate aminotransferase; ALT: alanine aminotransferase; LDH: Lactate dehydrogenase; BNP: B-type natriuretic peptide.

pathogenesis over a direct virus invasion-mediated tissue injury. Infection with COVID-19 triggers the formation of antibodies to viral surface epitopes. Virus neutralization is a direct function of the stochiometric concentration and affinity of the antibodies. It is believed that low titer non-neutralizing antibodies may accentuate virus triggered immune responses instead, thereby increasing the risk of severe illness in affected individuals [14]. While blocking antibodies against the angiotensin converting enzyme (ACE) receptor binding regions (such as the RBD and HR2 region of $\mathrm{S}$ protein) are deemed protective, those directed against nucleocapsid and other epitopes on $\mathrm{S}$ protein are not $[15,16]$. Weak antibody coated virus gets internalized by Fc receptors, followed by endosomal release of the virion and subsequent Tolllike receptor and cytosolic RNA sensor triggered IFN $\alpha$ responses. These antibody dependent enhancement (ADE) responses have been implicated in COVID-19 induced immune injury. Although evidence base for this pathway is demonstrated for coronaviruses [16], the exact role in PIMS-TS is only speculative [17].

\section{MANAGEMENT}

Conventionally, treatment of $\mathrm{KD}$ involves use of 
intravenous immunoglobulin (IVIG) and high dose aspirin as first line agents [18]. The use of IVIG for PIMS-TS may help in facilitating neutralization of virus and associated superantigens and downregulation of the inflammatory cytokines $[19,20]$. IVIG $(2 \mathrm{~g} / \mathrm{kg})$ has been used in most published series on PIMS-TS as first line therapy. The effects; however, may be short-lived $[9,10]$. In those with features of classic $\mathrm{KD}$, it would be appropriate to consider use of aspirin (30-50 mg/ $/ \mathrm{kg}$ /day followed by $3-5 \mathrm{mg} / \mathrm{kg} /$ day) along with IVIG [18]. The role of aspirin in children with hyperinflammation without features of KD is not known, and we believe that it has a limited role in these children. Although the role of anticoagulation is not clearly defined, it should be considered on a case-by-case basis in children with hyperinflammatory syndrome. The choice of anticoagulation and their dosing regimen would also depend on the presence of coronary aneurysms.

In select cases, especially those who do not respond to IVIG, adjunctive immunomodulatory therapy may be necessary to control inflammation. It is known that use of corticosteroids in $\mathrm{KD}$ is associated with earlier resolution of fever and lower incidence of coronary artery abnormalities [18,21]. Corticosteroids are also used as first line therapy in children with MAS. On this basis, it is plausible that these agents may be effective in PIMS-TS, especially in those with features of cytokine release syndrome (CRS). Recently published case series have shown that corticosteroids (initially pulse intravenous methylprednisolone $10 \mathrm{mg} / \mathrm{kg} /$ day for 3 days followed by oral prednisolone in a gradual tapering regimen) are useful adjuncts to IVIG in patients with PIMS-TS $[9,10,21]$.

Whilst not much is known about the pathogenesis of PIMS-TS, it is clear that there is elevation of cytokines such as IL-1, IL-6, IL-18 and IFN- $\alpha$ in most children who develop MAS [22]. Although this does not necessarily establish causality, specific cytokine blockade has resulted in remission of MAS on many occasions [23]. Also, specific blockade of TNF- $\alpha$ with infliximab has been tried in children with KD resistant to IVIG [18]. Along with IL-6, several other cytokine blockade therapies are currently under evaluation in adults with COVID-19. As we understand more about targeted therapy in adults with COVID induced CRS, we might consider trials of these agents in PIMS-TS [24,25]. Extrapolating these data, it is possible that there may be a role for specific cytokine blockade in PIMS-TS as well. Apart from one case report describing the use of tocilizumab in a child with KD and SARS-CoV-2 [6], data on use of biologics for this indication are still lacking. Until such data are available, it would be reasonable to consider these therapies only under special circumstances (in children with high CRP levels and those refractory to IVIG/corticosteroids) either in controlled clinical trials or by clinicians experienced in use of biologics. Where considered appropriate, therapy with biologics such as tocilizumab $(8 \mathrm{mg} / \mathrm{kg})$ or infliximab $(5 \mathrm{mg} / \mathrm{kg})$ should be considered. Based on existing evidence, suggested management of children with SARS-CoV-2 related hyperinflammation has been summarized in figure 1.

Apart from immunomodulation, supportive care plays a key role in the management of these children. Deterioration can be rapid, and it is important for clinicians to monitor for signs of worsening inflammation [4].

\section{FUTURE DIRECTIONS}

The important answers lie in understanding the immune origins of this condition. There is a need for clinical trials using adaptive designs (Bayesian methodology) which would enable us to evaluate therapies including IL-6, IL-1 and anti-TNF blockade in children with this syndrome.

\section{A. Supportive care \\ - Empirical antibiotics after obtaining blood cultures for suspected or evident bacterial infection. \\ - Intensive care support including vasopressors and assisted ventilation where indicated. \\ B. Specific management}

- Intravenous immunoglobulin $(2 \mathrm{~g} / \mathrm{kg})^{\star}$

- Consider aspirin (30-50mg/kg/d followed by $3-5 \mathrm{mg} / \mathrm{kg} / \mathrm{d}$ ) in those with classic Kawasaki disease.

Persistent inflammation

(Fever with raised inflammatory markers)

- Intravenous methylprednisolone (10mg/kg/day) for $3 \mathrm{~d}$

- Consider oral steroids (1-2mg/kg/d, weaning dose over $2 \mathrm{wk}$ ) for those with mild but persistent symptoms or signs of inflammation.

\begin{tabular}{|l|l|}
\multicolumn{1}{c|}{} & $\begin{array}{l}\text { Persistent inflammation } \\
\text { (Fever with very high inflammatory markers) }\end{array}$ \\
\hline Biologics & \\
- Tocilizumab $(8 \mathrm{mg} / \mathrm{kg})$ \\
- Infliximab $(5 \mathrm{mg} / \mathrm{kg})$
\end{tabular}

${ }^{*}$ Note: If IVIG is not available or is contraindicated, consider upfront use of corticosteroids; where possible, obtain blood samples for SARS-CoV-2 antibody testing or future research prior to administration of IVIG; Choice of anticoagulation and their dosing regimen would depend on presence of coronary artery aneurysms.

\#Only in centers with experience in use of biologics or in controlled clinical trials.

Fig. 1 Suggested management of SARS-COV-2 related hyperinflammation in children. 
Despite the emerging literature, there are still a lot of unknowns regarding SARS-CoV-2. It is important to gather data on the condition to understand the damage caused and risk for recurrence as well as long term implications including the risk for autoimmune disease later in life. Real time surveillance studies such as the WHO clinical data platform (https://apps.who.int/iris/ handle/10665/332236) and the British Pediatric Surveillance Unit (BPSU) study (https://www.rcpch.ac.uk/workwe-do/bpsu/study-multisystem-inflammatory-syndromekawasaki-disease-toxic-shock-syndrome) can gather information to help further our understanding of this disease. There is now an overwhelming need for registries for data collection and integration, especially in India [26,27]. Going forward, multicenter and perhaps multi-national collaborative studies may be required to fill existing gaps in our knowledge of the current pandemic and the new syndrome in children.

In the Indian context, we perceive a definite need for increased awareness of this unique clinical syndrome amongst parents and pediatricians alike in the midst of multitude of several common infections such as dengue, when a child presents with fever with variable accompanying symptoms and signs and raised inflammatory markers.

Contributors: CB, LG, AVR: substantial contribution to the conception and design of the work, preparation and finalization of the draft; CB, LG, SB, SS, AVR: substantial contributions to the acquisition, analysis, and interpretation of data for the work; SB, SS, AVR: Critical Revision for important intellectual content; CB, LG, SB, SS, AVR: final approval of the version to be published, and agreement to be accountable for all aspects of the work in ensuring that questions related to the accuracy or integrity of any part of the work are appropriately investigated and resolved.

Funding: None; Competing interests: None stated.

\section{REFERENCES}

1. Balasubramanian S, Rao NM, Goenka A, Roderick M, Ramanan AV. Coronavirus disease [COVID-19] in children - What we know so far and what we do not. Indian Pediatr. 2020; 57(5):435-442.

2. Meena J, Yadav J, Saini L, Yadav A, Kumar J. Clinical features and outcome of SARS-CoV-2 infection in children: A systematic review and meta-analysis. Indian Pediatr. 2020;S097475591600203. [published online ahead of print, 2020 Jun 24].

3. European Centre for Disease Prevention and Control. Pediatric inflammatory multisystem syndrome and SARSCoV-2 infection in children - 15 May, 2020. ECDC: Stockholm; 2020.

4. Royal College of Pediatrics and Child Health. GuidancePediatric multisystem inflammatory syndrome temporally associated with COVID-19, 2020. Available from: $h t t p s: / /$ www.rcpch.ac.uk/resources/guidance-pediatric-multi system-inflammatory-syndrome-temporally-associatedcovid-19. Accessed May 5, 2020.

5. Jones VG, Mills M, Suarez D, Hogan CA, Yeh D, Bradley SJ, et al. COVID-19 and Kawasaki disease: novel virus and novel case. Hosp Pediatr. 2020 Jun; 10(6): 537-540. [Epub ahead of print].

6. Balasubramanian S, Nagendran TM, Ramachandran B, Ramanan AV. Hyper-inflammatory syndrome in a child with COVID-19 treated successfully with intravenous immunoglobulin and tocilizumab. Indian Pediatr. 2020; S097475591600180. [E-pub ahead of print].

7. Multisystem inflammatory syndrome in children and adolescents with COVID-19. 15 May 2020 Scientific brief: World Health Organisation. Available from: https://www. who.int/publications-detail/multisystem-inflammatorysyndrome-in-children-and-adolescents-with-covid-19. Accessed May 31, 2020.

8. Centers for Disease Control and Prevention. Emergency preparedness and response: Health alert network. Published May 14, 2020. Available from: https:// emergency.cdc.gov/han/ 2020/han00432.asp. Accessed May 22, 2020.

9. Riphagen S, Gomez X, Gonzalez-Martinez C, Wilkinson $\mathrm{N}$, Theocharis P. Hyperinflammatory shock in children during COVID-19 pandemic. Lancet. 2020 May 23; 395 (10237):1607-1608.

10. Verdoni L, Mazza A, Gervasoni A, Martelli L, Ruggeri M, Ciuffeda M, et al. An outbreak of severe Kawasaki-like disease at the Italian epicentre of the SARS-CoV-2 epidemic: An observational cohort study. Lancet. 2020; 395: 1771-78.

11. Belhadjer Z, Méot M, Bajolle F, Khraiche D, Legendre A, Abakka S, et al. Acute heart failure in multisystem inflammatory syndrome in children [MIS-C] in the context of global SARS-CoV-2 pandemic. Circulation. 2020 May 17; CIRCULATIONAHA.120.048360. [E-pub ahead for print].

12. Tullie L, Ford K, Bisharat M, Watson T, Thakkar H, Mullassery D, et al. Gastrointestinal features in children with COVID-19: an observation of varied presentation in eight children. Lancet Child Adolesc Health. 2020;4: e19e20.

13. Whittaker E, Bamford A, Kenny J, et al. Clinical characteristics of 58 children with a pediatric inflammatory multisystem syndrome temporally associated with SARSCoV-2. JAMA. 2020; e2010369. [E-pub ahead for print].

14. Yu H, Sun B, Fang Z, Zhao J, Liu X, Li Y, et al. Distinct features of SARS-CoV-2-specific IgA response in COVID-19 patients. European Resp J. 2020; 2001526. [Epub ahead for print].

15. Iwasaki A, Yang Y. The potential danger of suboptimal antibody responses in COVID-19. Nature Reviews Immunology. 2020. Available from: http://www.nature. com/articles/s41577-020-0321-6. Accessed May 29, 2020.

16. Wang Q, Zhang L, Kuwahara K, Li L, Liu Z, Li T, et al. Immunodominant SARS coronavirus epitopes in humans elicited both enhancing and neutralizing effects on infection in non-human primates. ACS Infect Dis. 2016; 2(5):361-376. 
17. Shen L, Fanger MW. Secretory IgA antibodies synergize with IgG in promoting ADCC by human polymorphonuclear cells, monocytes, and lymphocytes. Cellular Immunology. 1981; 59(1):75-81.

18. McCrindle BW, Rowley AH, Newburger JW, Burns JC, Bolger AF, Gewitz M, et al. Diagnosis, treatment, and long-term management of Kawasaki disease: A scientific statement for health professionals from the American Heart Association. Circulation. 2017;135: e927-e99.

19. Burns JC, Franco A. The immunomodulatory effects of intravenous immunoglobulin therapy in Kawasaki disease. Expert Rev Clin Immunol. 2015;11:819-25.

20. Lo MS, Newburger JW. Role of intravenous immunoglobulin in the treatment of Kawasaki disease. Int J Rheum Dis. 2018;21:64-9.

21. Kobayashi T, Saji T, Otani T, Takeuchi K, Nakamura T, Arakawa $\mathrm{H}$, et al. Efficacy of immunoglobulin plus prednisolone for prevention of coronary artery abnormalities in severe Kawasaki disease [RAISE study]: A randomised, open-label, blinded-endpoints trial. Lancet. 2012; 379:1613-20.
22. Schulert GS, Grom AA. Pathogenesis of macrophage activation syndrome and potential for cytokine- directed therapies. Annu Rev Med. 2015; 66:145-59.

23. Schulert GS, Grom AA. Macrophage activation syndrome and cytokine-directed therapies. Best Pract Res Clin Rheumatol. 2014; 28:277-92.

24. Mehta P, McAuley DF, Brown M, Sanchez E, Tattersall RS, Manson JJ, et al. COVID 19: Consider cytokine storm syndromes and immunosuppression. Lancet. 2020; 395:1033-34.

25. Pacha O, Sallman MA, Evans SE. COVID-19: A case for inhibiting IL-17? Nat Rev Immunol. 2020; 20:345-46.

26. Acharyya BC, Acharyya S, Das D. Novel coronavirus mimicking kawasaki disease in an infant. Indian Pediatr. 2020; S097475591600184 [published online ahead of print, 2020 May 22].

27. Rauf A, Vijayan A, John ST, Hrishnan R, Latheef A. Multisystem inflammatory syndrome with features of atypical Kawasaki disease during COVID-19 pandemic. Indian J Pediatr. 2020; S12098020033571 [published online ahead of print, 2020 May 28]. 